Chapman University

Chapman University Digital Commons

ESI Working Papers

Economic Science Institute

$12-4-2018$

\title{
Using Response Times to Measure Ability on a Cognitive Task
}

\author{
Aleksandr Alekseev \\ ChapmanUniversity, alekseev@chapman.edu
}

Follow this and additional works at: https://digitalcommons.chapman.edu/esi_working_papers

Part of the Econometrics Commons, Economic Theory Commons, and the Other Economics Commons

\section{Recommended Citation}

Alekseev, A. (2018). Using response times to measure ability on a cognitive task. ESI Working Paper 18-16. Retrieved from https://digitalcommons.chapman.edu/esi_working_papers/254/

This Article is brought to you for free and open access by the Economic Science Institute at Chapman University Digital Commons. It has been accepted for inclusion in ESI Working Papers by an authorized administrator of Chapman University Digital Commons. For more information, please contactlaughtin@chapman.edu. 


\section{Using Response Times to Measure Ability on a Cognitive Task}

\section{Comments}

Working Paper 18-16

This working paper was later published as:

Alekseev, A. (2019). Using response times to measure ability on a cognitive task. Journal of the Economic Science Association. doi: 10.1007/s40881-019-00064-2 


\title{
Using Response Times to Measure Ability on a Cognitive Task*
}

\author{
Aleksandr Alekseev ${ }^{\dagger}$
}

December 4, 2018

\begin{abstract}
I show how using response times as a proxy for effort coupled with an explicit process-based model can address a long-standing issue of how to separate the effect of cognitive ability on performance from the effect of motivation. My method is based on a dynamic stochastic model of optimal effort choice in which ability and motivation are the structural parameters. I show how to estimate these parameters from the data on outcomes and response times in a cognitive task. In a laboratory experiment, I find that performance on a Digit-Symbol test is a noisy and biased measure of cognitive ability. Ranking subjects by their performance leads to an incorrect ranking by their ability in a substantial number of cases. These results suggest that interpreting performance on a cognitive task as ability may be misleading.
\end{abstract}

Keywords: cognitive ability, test scores, response times, drift-diffusion model, choice-process data

JEL codes: C24, C41, C91, D91, J24

${ }^{*}$ I thank Jim Cox, Glenn Harrison, Susan Laury, Tom Mroz, Vjollca Sadiraj, and Todd Swarthout for their valuable comments and suggestions. I thank conference participants at the Economic Science Association meetings, the Southern Economic Association meetings, and the Western Economic Association meetings, as well as seminar participants at Georgia State University, University of California San Diego, the University of Chicago, and Chapman University for their feedback. This work has been supported by the Andrew Young School Dissertation Fellowship.

${ }^{\dagger}$ Economic Science Institute, Chapman University, One University Drive, Orange, CA, 92866, e-mail: alekseev@chapman.edu, phone: +1 (714) 744-7083, ORCID: 0000-0001-6542-1920. 


\section{Introduction}

Economists become increasingly interested in using choice-process data in the empirical analysis. One of the earliest and arguably most popular examples of choice-process data is response times. The popularity of response times lies in the fact that collecting them is virtually costless given that most economic experiments are computerized while the potential benefits of having these data are large (Spiliopoulos and Ortmann, 2018). Researchers have been using response times in various ways, e.g., to study preferences, test models, and classify subjects. In this paper, I show how using response times as a proxy for effort coupled with an explicit process-based model can address a long-standing issue of how to separate the effect of cognitive ability on performance from the effect of motivation.

Correct measurement of cognitive ability is essential since ability is used as an explanatory variable in a vast array of contexts. Economists have been using cognitive ability to explain differences in earnings (Murnane et al., 1995; Heckman et al., 2006, 2013), risk and time preferences (Dohmen et al., 2010), the quality of decision-making (Agarwal and Mazumder, 2013), strategic reasoning (Gill and Prowse, 2016), as well as differences in various life outcomes, such as teenage pregnancy, marital status, smoking, and engaging in criminal activities (Duckworth et al., 2011). This literature traditionally uses performance on a cognitive test as a measure of cognitive ability. A fundamental flaw in this approach is that performance never reflects cognitive ability by itself. Performance also reflects character skills, such as motivation (Borghans et al., 2008; Duckworth et al., 2011; Segal, 2012). ${ }^{1}$ The traditional approach thus confounds actual ability with the combination of ability and motivation, which may result in wrong conclusions about the effect of ability. Using performance as a noisy proxy for ability could be justified if noise introduced by motivation is small relative to ability. However, the existing literature has little to say about the magnitude of the noise component in performance.

I propose a new approach to measure cognitive ability that overcomes the issues with the traditional approach. My method is based on a dynamic stochastic model of optimal effort choice

\footnotetext{
${ }^{1}$ For example, consider two students, Adam and Bob, who are taking a cognitive test. Adam has high cognitive ability but is not interested in the outcome of the test. Bob, on the other hand, has lower cognitive ability but is highly motivated to get the right answers. As a result, Bob might end up having a higher score on the test, which according to the traditional approach would imply that Bob has higher ability than Adam, while in reality, their ranking by ability is the opposite.
} 
in which ability and motivation are the structural parameters. I show how these parameters can be estimated from the data on outcomes and response times in a cognitive task. The proposed method is based on explicit modeling of the decision-making process and is inspired by the literature on drift-diffusion models (Ratcliff, 1978; Busemeyer and Townsend, 1993; Krajbich et al., 2012). These models have been shown to perform well in jointly predicting outcomes and response times, as well as to match the actual processes in the brain (Gold and Shadlen, 2007).

I use response times as a proxy for effort, following Wilcox (1993) and Ofek et al. (2007). An agent's effective effort is modeled as a Brownian motion with drift in which the drift rate represents the agent's ability. Higher ability leads to faster effort accumulation. The accumulated effective effort at a given time determines the probability to answer a question correctly. Correct answer yields utility that represents an agent's motivation. Effort is costly, and the more time an agent spends on a task, the higher will be the accumulated cost of effort. The agent's problem is to choose the optimal moment to stop the effort accumulation process. The solution to the agent's problem takes the form of a threshold rule in terms of the accumulated effective effort. I derive a closed-form solution for the optimal threshold and show how it is related to ability and motivation. The parameters of the model can be estimated using the maximum likelihood method using the data on outcomes and response times from a series of trials of a cognitive task. The proposed estimation strategy can be viewed as a version of a threshold regression model used in survival analysis (Lee and Whitmore, 2006).

I conduct a laboratory experiment to illustrate the proposed approach and compare it to the traditional approach. In the experiment subjects take a Digit-Symbol test (DST) in which they have to match symbols to digits. DST is designed to capture a subject's processing speed, which underlies more complex cognitive functions. DST is used in the economics literature (Segal, 2012; Dohmen et al., 2010)) and in intelligence scales such as WAIS (Weiss et al., 2010). Subjects are free to choose how much time to spend on a task and are not extrinsically motivated for good performance. I estimate ability and motivation for each subject individually and use the structural model to perform a counterfactual simulation in which the only source of variation in performance is variation in ability. I find that performance is a noisy and biased measure of ability. Variation in ability can explain only 0.58 of the variation in observed performance. Subjects with relatively low ability have lower performance than they would have if performance were an unbiased measure of 
ability, while subjects with relatively high ability have even higher performance than they would have. Ranking subjects by performance leads to an incorrect ranking by ability $24 \%$ of the time.

These results suggest that more care should be given when interpreting performance as cognitive ability since such an interpretation may be misleading. The present paper, however, should be viewed as a first step towards uncoupling ability from motivation on performance. More work is needed to understand how well performance approximates ability in other cognitive and real-effort tasks used in the literature. The main goal of the present paper is to provide the tools for this work and to illustrate the usefulness of choice-process data and process-based modeling in developing such tools.

\section{Theoretical Model}

Consider an agent working on a trial of a cognitive task. An outcome of the trial can be either a success (the answer given by the agent is correct) or a failure (the answer given by the agent is incorrect). The agent can exert effort to increase the probability of success, which is a measure of the agent's performance. Following the literature on the drift-diffusion model, I assume that the agent accumulates effort according to the Brownian motion with drift:

$$
d E_{t}=\alpha d t+\sigma d W_{t}
$$

in which the drift rate $\alpha>0$ represents the agent's ability and the diffusion parameter $\sigma>0$ represents her (inverse of) consistency. Ability in this model is equivalent to the efficiency of converting effort (time spent on a trial) into performance (probability of success). ${ }^{2}$ Given a fixed amount of time, an agent with higher ability will have higher performance on the task than an agent with lower ability. Having higher ability in the model thus corresponds well to an intuitive notion of being good, or able, at doing something.

The agent stops effort accumulation and gives an answer to a trial when the effort process hits a threshold. Unlike in a typical drift-diffusion model, I assume that there is a single threshold that is

\footnotetext{
${ }^{2}$ The model assumes that response time is the only margin of effort and that agents do not differ in their effort intensity.
} 
chosen optimally by the agent. The agent choose the threshold by maximizing her utility function

$$
\mathbb{E}\left[\int_{0}^{\tau}-e^{-\rho t} d t+\mu p\left(E_{\tau}\right) e^{-\rho \tau}\right]
$$

in which the first part is the accumulated cost of effort by the time $\tau$ and the second part is the expected benefit from a success on a trial. The agent uses a discount rate $\rho>0$ and is assumed to experience a unit cost of effort, in utility terms, per unit of effort (time in this case) spent on a trial. The utility of success is $\mu>0$, and the utility of failure is normalized to zero. Utility $\mu$ represents the agent's motivation for succeeding on a task. ${ }^{3}$ This motivation is determined by a combination of the agent's character and the characteristics of a task. The probability of success $p(\cdot)$ depends on the accumulated effort by the time $\tau$.

To solve the agent's problem analytically, I assume that $p(E)=1-e^{-E}$. The optimal threshold for the agent's problem is then

$$
E^{*}(\alpha, \mu, \sigma, \rho)=\ln \left(1+\frac{1}{\beta}\right)-\ln \left(1+\frac{1}{\mu \rho}\right), \text { where } \beta \equiv \frac{-\alpha+\sqrt{\alpha^{2}+2 \rho \sigma^{2}}}{\sigma^{2}}
$$

The agent's optimal threshold, and hence her performance, is increasing in ability. The effect of ability on average effort, $\bar{t}=\frac{E^{*}}{\alpha}$, cannot be unambiguously signed. In general, this effect depends on the particular values of ability and motivation.

\section{Estimation Strategy}

Suppose that we observe a sequence of $N$ independent and identical trials of a cognitive task performed by an individual. Each observation is a pair $\left(x_{i}, t_{i}\right), i=1, \ldots, N$, where $x_{i}$ is an outcome of a trial $i$ and $t_{i}$ is the response time in that trial. The likelihood of an observation $i$, conditional on the parameters of the model $\theta \equiv(\alpha, \mu, \sigma)$, is ${ }^{4}$

$$
l\left(x_{i}, t_{i} \mid \theta\right)=p\left(E^{*}(\theta)^{x_{i}}\left(1-p\left(E^{*}(\theta)\right)^{1-x_{i}} f\left(t_{i} \mid \theta\right)\right),\right.
$$

\footnotetext{
${ }^{3}$ Strictly speaking, motivation in this model is measured in the units of cost effort.

${ }^{4}$ For simplicity, I assume that the discount rate is known and is the same for each individual.
} 
where the first part is simply the Bernoulli likelihood. The second part, $f\left(t_{i} \mid \theta\right)$, is the likelihood that the stochastic process $(1)$ hits the threshold $E^{*}$ at time $t_{i}$. In particular, for the process that starts at zero, this likelihood is

$$
f(t \mid \theta)=\frac{E^{*}(\theta)}{\sqrt{2 \pi \sigma^{2} t^{3}}} \exp \left(-\frac{\left(E^{*}(\theta)-\alpha t\right)^{2}}{2 \sigma^{2} t}\right)
$$

The ability of each individual, as well as the two other parameters of the model, can then be estimated using the maximum likelihood method:

$$
\hat{\theta}=\underset{\theta}{\arg \max } \ln \mathcal{L}(\theta \mid \mathbf{x}, \mathbf{t}) \equiv \sum_{i=1}^{N} \ln l\left(x_{i}, t_{i} \mid \theta\right) .
$$

The identification of the model can be shown using the following logic. First, the data on the outcomes of trials allow one to infer what was the probability of success. This probability remains constant in every trial. Second, the mapping of the optimal threshold into the probability of success allows one to infer what was the optimal threshold in each trial. Third, the data on response times combined with the inferred optimal threshold pins down the values of ability and consistency. Finally, the inferred optimal threshold together with the inferred values of ability and consistency, and the mapping from the parameters into the optimal threshold, pin down the remaining unknown parameter: motivation.

\section{Experiment}

To illustrate the method, I conducted an experiment at the Experimental Economics Center lab at Georgia State University (GSU) in June 2017 and March-April 2018. The experiment consists of 11 sessions with 192 participants in total. The subjects in the experiment are undergraduate students at GSU. The average earnings in the experiment are $\$ 36.35$.

The main part of the experiment is a cognitive task, which is a version of a Digit-Symbol test (DST). ${ }^{5}$ In a DST, subjects have to find correct correspondences between digits and symbols. In the present implementation, subjects are given a key with six digit-symbol pairs and a list of 14

\footnotetext{
${ }^{5}$ The experiment also included a risk elicitation task and a survey, results of which are not reported here.
} 
symbols to fill six numbered boxes. ${ }^{6}$ The DST consists of 100 trials in which the key and the list of available symbols change in every trial. ${ }^{7}$ Subjects are free to choose how much time to spend on each trial. ${ }^{8}$ Unconstrained (or endogenous, in the language of Spiliopoulos and Ortmann (2018)) response time is important in this context since response time is assumed to be the only margin of effort in the experiment. In order to minimize the interdependency between the rounds (e.g., via learning) as much as possible, I do not provide subjects with any feedback between the rounds. Subjects learn their score only at the end of the experiment. The incentives in the DST are flat: each subject receives $\$ 20$ for completion regardless of performance. This incentive scheme allows one to elicit a subject's intrinsic motivation since good performance is not extrinsically incentivized.

The benefit of a DST is that it measures fluid intelligence, i.e., the ability to solve novel problems that do not rely on any cultural background or accumulated knowledge for solution (Cattell, 1971). Performance on a DST is associated with processing speed. Processing speed is positively associated with other IQ measures since the processing speed is the basis for more complex cognitive functions (Vernon, 1983). In economics, researchers have used a DST to study the relationship between cognitive ability and risk and time preferences (Dohmen et al., 2010) and the role of motivation in performance (Segal, 2012).

\section{Results}

Subjects perform surprisingly well on the DST given that they were not extrinsically rewarded for good performance. The median score is 92 and the interquartile range (IQR) for the score is only 9. Such high scores suggest that the subjects had non-trivial levels of intrinsic motivation in the task. The median subject took 20.6 seconds on average to complete a single trial. The IQR for the mean response time (MRT) is 6.9. To get a rough idea of processing speed, one can look at the

\footnotetext{
${ }^{6}$ See Appendix A for the subject instructions and screenshots.

${ }^{7}$ In a traditional implementation of a DST, the key does not change across trials. Performance on a traditional DST then captures subjects' working memory in addition to processing speed. In the present context, however, processing speed is the only quantity of interest. See Benndorf et al. (2018) for a similar argument.

${ }^{8}$ In most implementations, the time that is allowed to spend on a cognitive task is constrained. The performance measure that is used in the present experiment, i.e., performance with no time constraint, is, therefore, not strictly identical to the performance measures typically used. The underlying message, however, would remain the same even if the time were constrained: in order to separate the effect of ability from the effect of motivation, one needs to supplement a measure of performance with a measure of effort. In the case of a time constraint, however, the relevant measure of effort would be difficult to observe.
} 
Figure 1: Distributions of Raw and Transformed Ability
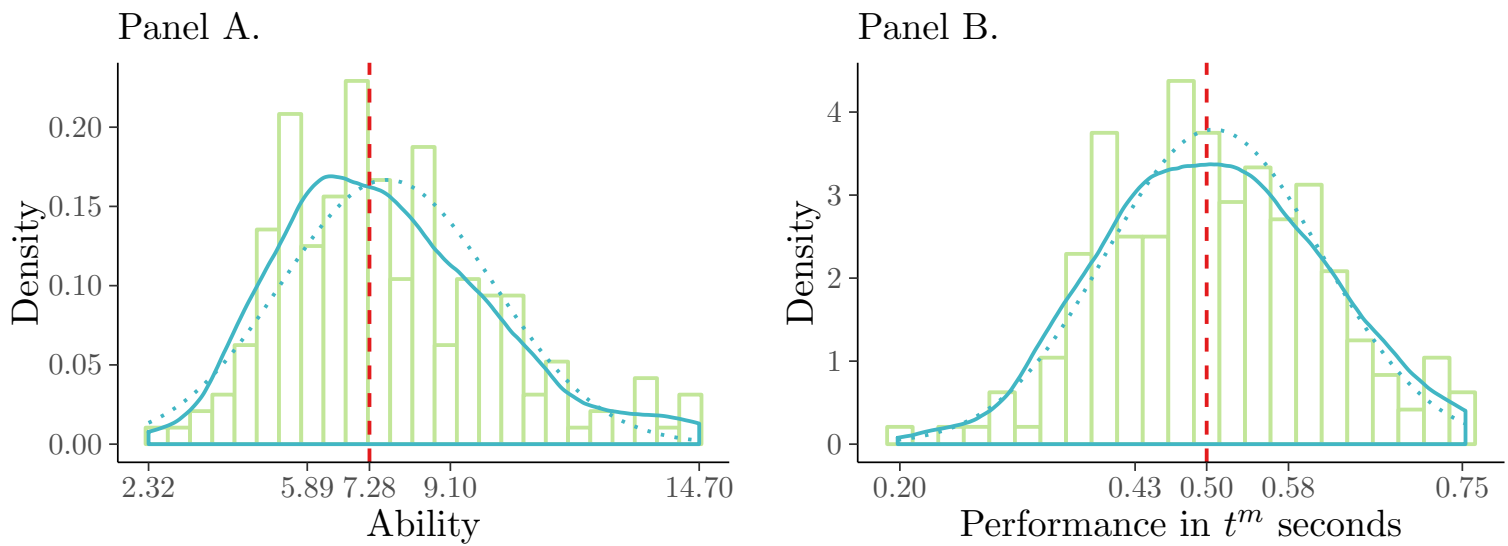

Note: Panel A shows the distribution of the individual-level estimates of ability in the sample. Panel B shows the distribution of the probability of success in $t^{m}$ seconds. The smooth solid line is the kernel density estimate, the vertical bars are the histogram, the dotted line is the reference density of a normal distribution with the parameters matching the sample moments, and the vertical dashed line is the sample median. The breaks on the horizontal axis correspond to the quintiles of the distribution.

ratio of a score to total time spent on a task. According to this measure, the median subject gave 2.6 correct answers per minute.

Figure 1 (Panel A) shows the distribution of the individual-level estimates of the ability parameter $\alpha .{ }^{9}$ The distribution is bell-shaped and concentrated around the median but asymmetric. The sample distribution has a higher mass of subjects with a just-below-median ability and a longer and fatter right tail relative to a reference normal distribution. The graph shows considerable variation in ability among subjects. For example, a subject at the 75th percentile is 1.5 times better at converting their exerted effort into accumulated effective effort than a subject at the 25th percentile. A subject with the highest ability is 2 times better than a median subject and 6.3 times better than a subject with the lowest ability.

These differences in ability become muted when one considers how they translate into differences in performance. This is expected since the probability-of-success function is a concave transformation of the accumulated effective effort. To translate the estimates of ability into performance, I compute the probability of success at the average accumulated effective effort in $t^{m}$ seconds, where $t^{m}(\approx 5.71$ seconds $)$ is calibrated such that it is the time for a person with median ability to reach a

\footnotetext{
${ }^{9}$ The subjects with a perfect score of 100 (6 subjects or $3 \%$ of the sample) were assigned a score of 99 by randomly selecting a trial and assigning it as incorrectly solved. The model cannot be estimated in the case of a perfect score. Excluding the subjects with perfect scores does not alter the results significantly.
} 
0.5 probability of success. Due to variation in ability, subjects will have different levels of accumulated effective effort in $t^{m}$ seconds, which will then translate into different probabilities of success. Panel B on Figure 1 shows the distribution of the resulting performance. This distribution is more symmetric than the distribution of ability estimates. In fact, one cannot reject the null hypothesis of the distribution of performance in $t^{m}$ seconds being normal (Shapiro-Wilk test $p$-value $=0.574$ ). A subject at the 75 th percentile would have a 1.4 times higher performance in $t^{m}$ seconds than a subject at the 25 th percentile. A subject with the highest ability would have a 1.5 times higher performance than a median subject and a 3.8 times higher performance than a subject with the lowest ability.

The method of converting estimated ability into performance described above helps to understand what differences in ability mean for subjects' performance. However, this method is purely mechanical in the sense that it does not take into account subjects' optimal choices and thus is not suitable for comparison with the observed performance. To overcome these issues, I use formula (3) to compute the optimal effort threshold, as well as the probability of success implied by this threshold, for each subject while holding the values of motivation and consistency fixed at the median level. This procedure yields the distribution of counterfactual performance that would arise in the sample due to variation in ability alone. Note that this counterfactual performance is stripped down from all the variation in motivation and consistency and thus can be used as a valid measure of ability.

Before comparing the observed and counterfactual performance, it is worth to recall a simple model of performance as a noisy measure of the true underlying ability:

$$
P_{1}=P_{0}+\epsilon,
$$

where $P_{1}$ is the observed performance, $P_{0}$ is the counterfactual performance defined as above that reflects the true underlying ability, ${ }^{10}$ and $\epsilon$ is a mean-zero noise term. Noise in this model is caused by the variation in motivation and consistency. For the observed performance to be a good proxy for ability, the variance of the noise $\sigma_{\epsilon}^{2}$ should be small relative to the variance of the observed

\footnotetext{
${ }^{10}$ For simplicity, I assume that there is no measurement error in the counterfactual performance, which of course will not be true in practice. Allowing for this additional measurement error would only increase the overall noisiness of the observed performance.
} 
Figure 2: Observed and Counterfactual Performance

Panel A.

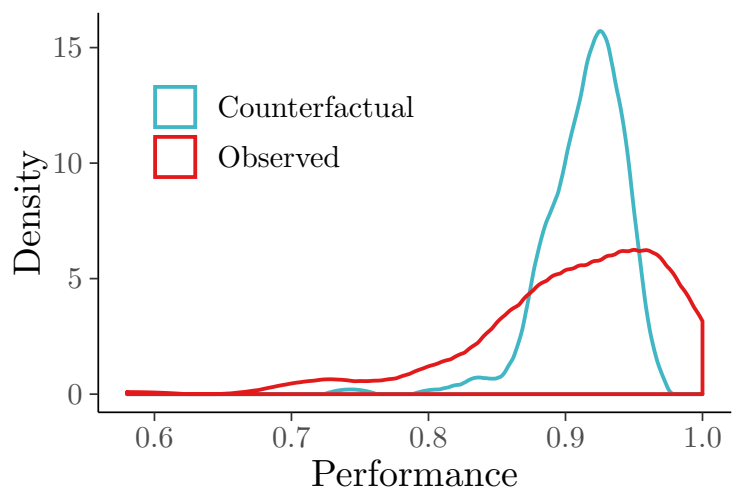

Panel B.

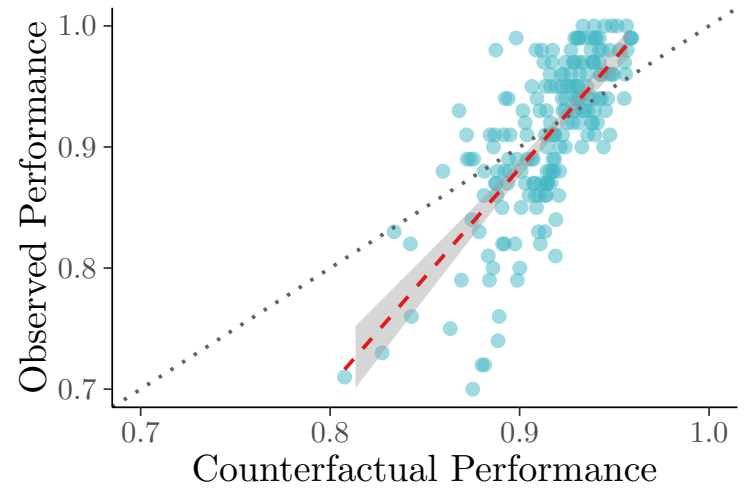

Note: Panel A shows the kernel density estimates of the distributions of observed and counterfactual performance. Panel B shows the scatterplot of the observed and counterfactual performance. The dotted line is the 45-degree line. The dashed line is the linear fit.

performance $\sigma_{P_{1}}^{2}$, and the noise term should be orthogonal to ability, $\operatorname{cov}\left(P_{0}, \epsilon\right)=0$. The observed performance in our case fails to satisfy either property.

Figure 2 (Panel A) plots the kernel density estimates of the distributions of the observed and counterfactual performance. It is immediately clear that the observed performance contains a substantial degree of noise. The ratio of the variance of the noise to the variance of the observed performance, $\sigma_{\epsilon}^{2} / \sigma_{P_{1}}^{2}$, is 0.55 . Such a high noise component results in only a moderate association between the observed and counterfactual performance: variation in the counterfactual performance can explain only 0.58 of the variation in the observed performance in a simple linear regression. If one cares only about whether the ranking by performance is similar to the ranking by ability, the picture is similarly unsatisfactory (Kendall's $\tau=0.54, p$-value $<0.001$ ). In particular, ranking subjects by their performance leads to an incorrect ranking by their ability in $24 \%$ of cases. $^{11}$

However, the observed performance is not just a highly noisy measure of ability. It is also a biased measure. The issue lies in the fact that ability, as measured by the counterfactual performance, is positively associated with the noise term (Kendall's $\tau=0.28, p$-value $<0.001$ ). Panel B on Figure 2 illustrates the bias that results from this association by presenting a scatterplot of the observed performance against the counterfactual performance. If the observed performance were an unbiased measure of true ability, the dots on the graph would lie along the 45-degree line (dotted

\footnotetext{
${ }^{11} \mathrm{An}$ alternative way to interpret this number is that the probability that two subjects taken at random will have incorrect ability ranking, as implied by performance, is $24 \%$.
} 
line on the graph). This is clearly not the case. Subjects with relatively low ability $(<0.92)$ score less than they should, while subjects with relatively high ability $(>0.92)$ score even higher than they should. This bias is represented by a linear fit (dashed line on the graph) that has a slope greater than one and a negative intercept.

The high degree of noise coupled with the systematic bias in the observed performance is likely to lead to invalid inferences when ability, proxied by performance, is used as a control or a causal regressor. In particular, one might find a positive association between performance and some outcome of interest and thus conclude that this outcome is related to ability, while in reality ability and the outcome are unrelated. When performance is used as a control variable, one may find that it is not significant and wrongly conclude that ability does not change the relation between the outcome of interest and a causal variable.

\section{Conclusion}

The economics literature uses cognitive ability as an explanatory variable in a vast array of economic contexts. The traditional approach of using performance on a cognitive test as a measure of ability confounds actual ability with the combination of ability and motivation, which may result in wrong conclusions about the effect of ability. In this paper, I propose a new approach to measure cognitive ability that overcomes this issue. The proposed approach is based on using response times data, in addition to performance data, as a proxy for effort together with an explicit process-based model inspired by the drift-diffusion model. I model ability and motivation as parameters of the structural model and show how to estimate these parameters from the data on outcomes and response times in a cognitive task. In a laboratory experiment, I find that performance is a noisy and biased measure of cognitive ability. Ranking subjects by their performance leads to an incorrect ranking by their ability in a substantial number of cases. These results suggest that more care should be given when interpreting performance as cognitive ability, as is usually done, since such an interpretation may be misleading. Future work should investigate how well performance approximates ability in other cognitive and real-effort tasks used in the literature. 


\section{Appendices}

\section{A Experimental Instructions}

This task is based on finding correct correspondences between numbers and symbols. In each round, you will see 6 pairs of number-symbol combinations (the key) arranged in a table at the upper part of the screen, see Figure A.1a for an example. Below the key, there will be 6 empty numbered boxes.

You will use the key to fill in the boxes with the symbols located in a column to the left of the boxes. You will do this by dragging the symbols into the boxes. If a symbol from the column is in the key, drag it to the corresponding numbered box. Some of the symbols will not be listed in the key. In this case, you should not use them in any of the boxes. Some of the numbers will not have corresponding symbols. In this case, you should leave those boxes empty. Each box, therefore, can contain either one or no symbols. Figure A.1b shows an example of a correctly solved round.

After filling all the boxes as you see fit, click "Submit", and you will proceed to the next round. You can proceed with each round at your own pace, there is no time limit. We ask that you complete all 100 rounds of the task. We will show you your score at the end of the task. You will receive $\$ 20$ for completing this task.

You will have 3 practice rounds before the actual task begins. This will give you a chance to familiarize yourself with the interface. During the practice, you will receive feedback if you make a mistake.

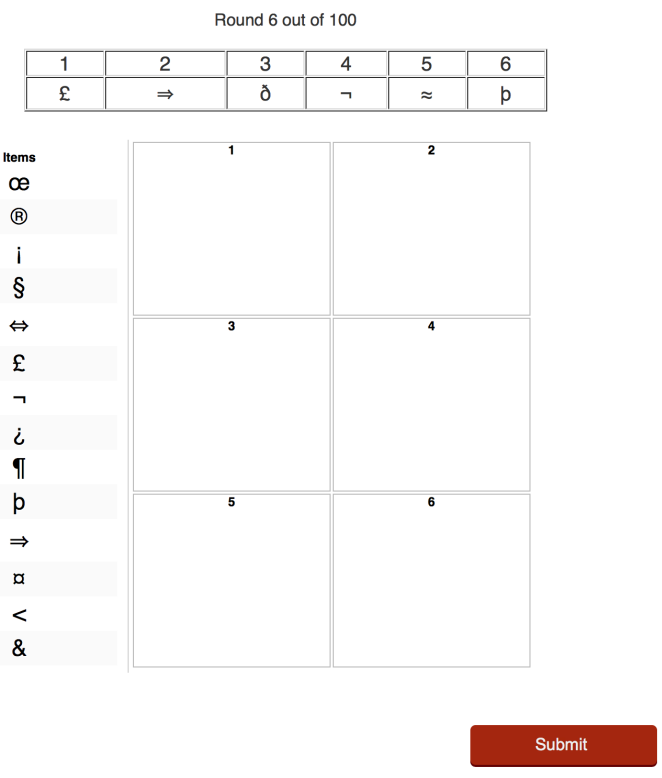

(a) Decision screen

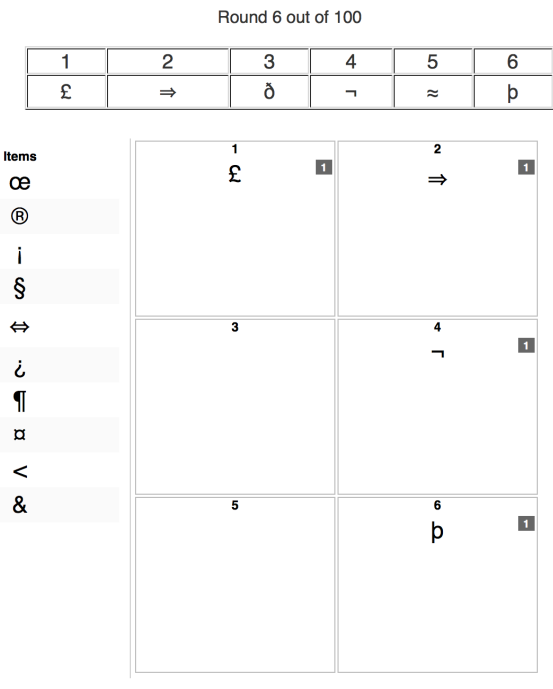

Submit

(b) Correct solution

Figure A.1: Digit-Symbol Task 


\section{References}

Agarwal S, Mazumder B (2013). "Cognitive Abilities and Household Financial Decision Making." American Economic Journal: Applied Economics, 5(1), 193-207.

Benndorf V, Rau HA, Sölch C (2018). "Minimizing Learning Behavior in Repeated Real-Effort Tasks." Working Paper 343, Center for European, Governance and Economic Development Research, Georg-August-Universität Göttingen.

Borghans L, Duckworth AL, Heckman JJ, Ter Weel B (2008). "The Economics and Psychology of Personality Traits." Journal of Human Resources, 43(4), 972-1059.

Busemeyer JR, Townsend JT (1993). "Decision Field Theory: A Dynamic-Cognitive Approach to Decision Making in an Uncertain Environment." Psychological Review, 100(3), 432.

Cattell RB (1971). Abilities: Their Structure, Growth, and Action. Boston: Houghton Mifflin.

Dohmen T, Falk A, Huffman D, Sunde U (2010). "Are Risk Aversion and Impatience Related to Cognitive Ability?" American Economic Review, 100(3), 1238-1260.

Duckworth AL, Quinn PD, Lynam DR, Loeber R, Stouthamer-Loeber M (2011). "Role of Test Motivation in Intelligence Testing." Proceedings of the National Academy of Sciences, 108(19), $7716-7720$.

Gill D, Prowse V (2016). "Cognitive Ability, Character Skills, and Learning to Play Equilibrium: A Level-k Analysis." Journal of Political Economy, 124(6), 1619-1676.

Gold JI, Shadlen MN (2007). "The Neural Basis Of Decision Making." Annual Review of Neuroscience, 30, 535-574.

Heckman J, Pinto R, Savelyev P (2013). "Understanding the Mechanisms Through Which an Influential Early Childhood Program Boosted Adult Outcomes." American Economic Review, 103(6), 2052-2086.

Heckman JJ, Stixrud J, Urzua S (2006). "The Effects of Cognitive and Noncognitive Abilities on Labor Market Outcomes and Social Behavior." Journal of Labor Economics, 24(3), 411-482.

Krajbich I, Lu D, Camerer C, Rangel A (2012). "The Attentional Drift-Diffusion Model Extends to Simple Purchasing Decisions." Frontiers in psychology, 3, 193.

Lee MLT, Whitmore G (2006). "Threshold Regression for Survival Analysis: Modeling Event Times by a Stochastic Process Reaching a Boundary." Statistical Science, pp. 501-513.

Murnane R, Willett JB, Levy F (1995). "The Growing Importance of Cognitive Skills in Wage Determination." The Review of Economics and Statistics, 77(2), 251-66.

Ofek E, Yildiz M, Haruvy E (2007). "The Impact of Prior Decisions on Subsequent Valuations in a Costly Contemplation Model." Management Science, 53(8), 1217-1233.

Ratcliff R (1978). "A Theory of Memory Retrieval.” Psychological Review, 85(2), 59.

Segal C (2012). "Working When No One Is Watching: Motivation, Test Scores, and Economic Success." Management Science, 58(8), 1438-1457. 
Spiliopoulos L, Ortmann A (2018). "The BCD of Response Time Analysis in Experimental Economics." Experimental Economics, 21(2), 383-433.

Vernon PA (1983). "Speed of Information Processing and General Intelligence." Intelligence, 7(1), $53-70$.

Weiss LG, Saklofske DH, Coalson DL, Raiford SE (2010). WAIS-IV Clinical Use and Interpretation: Scientist-Practitioner Perspectives. Academic Press.

Wilcox NT (1993). "Lottery Choice: Incentives, Complexity and Decision Time." The Economic Journal, 103(421), 1397-1417. 